\title{
Special issue on Intelligence Computation Evolutionary Computation: ICEV2018
}

\author{
Zhenyu $\mathrm{Du}^{1}$
}

Published online: 31 July 2019

○) Springer-Verlag GmbH Germany, part of Springer Nature 2019

This editorial describes a special issue of papers from the 2018 International Conference on Intelligence Computation Evolutionary Computation. There are 8 papers in this special issue.

Research on periodic disturbance suppression method in printer paper folding mechanism based on disturbance observer algorithm establishes a model of the system with MATLAB system identification tools in the process of establishing the control system, inverses this established model. In order to approximate the mode and guarantee the stability of the inversed model, zero-phase error-tracking controller (ZPETC) has been designed to remove the instable zeropoint of the original model. The paper constructs the disturbance observer of the established reversed model based on PID control algorithm.

A polychromatic sets theory based algorithm for the input/output scheduling problem in AS/RSs proposes a hybrid polychromatic sets theory based genetic algorithm (GA) for the input/output scheduling problem of automated storage and retrieval systems (AS/RSs). In order to overcome the drawbacks of precocious phenomena and lack of stability of the GA, a novel hybrid model through integration of genetic algorithm (GA) and polychromatic sets theory (PS) is proposed. During the solution process, polychromatic sets contour matric is used to assign input/output goods location reasonably to improve the quality of initial population. During the iterative process, simulate anneal algorithm (SA) is invoked to jump out of the local optimum to obtain a satisfactory solution.

Imbalanced data classification algorithm with support vector machine kernel extensions proposes a imbalanced data classification algorithm of support vector machines (KE-SVM). This algorithm achieve the initial classification

Zhenyu Du

itie@vip.163.com

1 Information Technology and Industrial Engineering

Research Center, Hong Kong, China of data samples by training the maximum margin classification SVM model, and then obtaining a new kernel extension function. based on Chi square test and weight coefficient calculation, through training the samples again by the new vector machine with kernel function to improve the classification accuracy. Through the simulation experiments of real data sets of artificial data set, it shows that the proposed method has higher classification accuracy and faster convergence for the uneven distribution data.

Removal of rain video based on temporal intensity and chromatic constraint of raindrops proposes an improved algorithm of frame time difference. This paper analyzes the temporal intensity waveform and chromatic constraint properties of raindrops, and the method is optimized by these two properties. We make use of the difference between rain and non-rain moving objects in the pixels' intensity changes, which realized a broad classification between the rain and non-rain moving object pixel. The candidate raindrops pixels are optimized in combination with the chromatic constraint property. The experimental results show that the proposed algorithm has a better effect of rainy day in video image restoration than Garg's, and it is simple and effective. The algorithm has a strong applicability, and it can be further used for many applications, such as air pollution control, management, outdoor surveillance, remote sensing and intelligent vehicles.

A gas source localization algorithm based on NLS initial optimization of particle filtering propose a gas source localization algorithm based on the initial NLS value optimization of particle filter (PF-NLS). Firstly, the state space model of the system is established by using the gas turbulence diffusion model, the particle weight is updated by constructing the likelihood function, and then the initial position and initial source strength of the gas source are obtained by using the NLS algorithm. Finally, the true information of the gas source is accurately estimated by using the PFNLS algorithm. The simulation results show that compared with NLS, the algorithm has higher positioning accuracy, 
further improves the convergence speed of the algorithm by optimizing the initial value, and can be well applied to practical scenarios.

FPGA logic design method based on multi resolution image real time acquisition system proposes a scheme of real-time and multi resolution image acquisition system by FPGA control logic design based on the real-time data acquisition, beam forming, processing, acquisition and transmission to ARM embedded video signal processing system with high real-time requirements, so we choose FPGA and SRAM real-time acquisition system which can meet the above requirements in speed and capacity. According to the problems encountered in the process of high speed acquisition, a reasonable timing optimization and RTL level synthesis is designed. The experimental results show that the design and implementation of the image acquisition system is not only stable, but also significantly improve the overall performance of the image processing system.

Research on cloud computing in the resource sharing system of university library services builds cloud library platform architecture, introduces the cloud computing related technology, such as Hadoop. The building of the library constitutes a local library cloud platform uses Web technology to achieve the scheduling of local libraries, and then implements the most common resource retrieval service in the library on the cloud platform framework, and finally discusses issues that need attention in the follow-up development of the platform. A multi-agent sharing model for educational information resources under the cloud-computing environment has established to serve the construction of library information resources better. In addition, this article innovatively puts forward the function of library construction of employment information services for college students, which is better to serve college students.

Short-term electrical load forecasting method based on stacked auto-encoding and GRU neural network proposes a short-term electrical load forecasting method based on stacked auto-encoding and GRU (Gated recurrent unit) neural network. Firstly, the method input historical data which contains power load, weather information, and holiday information, and use auto-encoding to compress the historical data; and then, the multi-layer GRU is used to construct the model to predict the power load. The experiment results show, compared with traditional models, the proposed method can effectively predict the daily variation of power load and have lower prediction error and higher precision.

We are grateful to the authors who submitted papers to this special issue. We would also like to thank the reviewers for their hard work and their valuable feedback to the authors. Finally, we would like to express our sincere gratitude to the Editor in Chief, for providing the opportunity and assistance to edit this special issue in the international journal of Evolutionary Intelligence.

Publisher's Note Springer Nature remains neutral with regard to jurisdictional claims in published maps and institutional affiliations. 